\title{
Predicting sentencing outcomes with centrality measures
}

\author{
Carlo Morselli ${ }^{1 *}$, Victor Hugo Masias ${ }^{2,3}$, Fernando Crespo $^{4}$ and Sigifredo Laengle ${ }^{3}$
}

\begin{abstract}
Despite their importance for stakeholders in the criminal justice system, few methods have been developed for determining which criminal behavior variables will produce accurate sentence predictions. Some approaches found in the literature resort to techniques based on indirect variables, but not on the social network behavior with exception of the work of Baker and Faulkner [ASR 58: 837-860, 1993]. Using information on the Caviar Network narcotics trafficking group as a real-world case, we attempt to explain sentencing outcomes employing the social network indicators. Specifically, we report the ability of centrality measures to predict a) the verdict (innocent or guilty) and b) the sentence length in years. We show that while the set of indicators described by Baker and Faulkner yields good predictions, introduction of the additional centrality measures generates better predictions. Some ideas for orienting future research on further improvements to sentencing outcome prediction are discussed.
\end{abstract}

Keywords: Criminology, Sentencing outcomes, Social networks

\section{Introduction}

This study examines the prediction of criminal trial sentencing outcomes on the basis of social network measures. Though it has received relatively little attention in criminology, sentencing predictions are extremely important to various stakeholders in the criminal justice system [1,2]. In general terms, law enforcement entities are responsible for three central tasks: a) monitoring, b) making arrests, and c) charging one or more persons [2]. In their pursuit of these activities, however, they normally do not have the necessary data and methods at their disposal to identify which individual and group characteristics influence the fate of those they lay charges against. Furthermore, attempts at prediction are complicated by the fact that judicial processes are not free of bias due to discrimination or errors stemming from the lack of standard sentencing guidelines [3-7].

While studies aimed at specifying the factors influencing criminal conduct may be found in past research [8], few focus on explaining sentencing outcome based on the networking features of offenders. Research has concentrated rather on explaining outcomes using sociodemographic and socioeconomic variables. For example, sentences have

\footnotetext{
* Correspondence: carlo.morselli@umontreal.ca

'School of Criminology, Université de Montréal, C.P. 6128, succursale Centre-ville, Montreal, QC H3C-3 J7, Canada

Full list of author information is available at the end of the article
}

been shown to fluctuate in accordance with political environment indicators [9], an individual's race and age [10,11], and an individual's criminal history and the presence of a police confession [12].

Our concern is to access sentencing outcomes as a function of behavior and positioning in criminal networks in order to determine whether the judicial processes that define sentences capture and take this behavior into account. From a social network perspective, networks of nodes represent individuals (or actors) and the direct and indirect relationships between them [13]. As Sarnecki has noted: "One of the most important tasks of network analysis is to attempt to explain, at least in part, the behavior of the elements in a network by studying specific properties of the relations between these elements" [14] p. 5. This method is already established as a powerful tool in many fields such as marketing, political science, organizational behavior, epidemiology, sociology, and software development [15-17]. Other theoretical and empirical initiatives have extended its use to the analysis of the social behavior of criminal groups and organizations $[2,18-20]$ and terrorists operations [21,22].

A key aspect of the social network approach was pointed to by McGloin and Kirk: "[n]etwork analysis requires different data than most criminologists typically employ. It may be clear by now that the unit of analysis in network 
studies is not the node or individual, but the tie between entities (i.e., links among the nodes)" [23], p. 212. More specifically, this approach allows us to identify a) the central individuals in a criminal network, b) its subgroups, c) interactions between the subgroups, d) the network's structure, e) the impact of removing an individual from the network, and f) the network's information flows $[20,23]$. It also affords a broad array of measures and technologies from various generations that facilitate the study of organized criminal behavior [24]. In short, network analysis provides both a theoretical basis and practical methods criminologists can employ to study the variables that explain criminal behavior and the interpersonal relationships underlying it.

Most existing studies of criminal networks focus on characterizing the roles of individual members, especially those whose roles are central $[1,20,23,25]$ or who serve as brokers [18,26-31]. Yet despite the growing popularity among criminologists of these social network applications, their practical usefulness is still limited [32]. The state of the art suggests there is little information regarding which social network variables can predict who will or will not be arrested or convicted and how long a sentence will be. One of the few works that explores the explanatory ability of certain social network and sentencing outcome measures is Baker and Faulkner [1]. The authors use various centrality indicators defined in Freeman [33] to explore the capacity of social network metrics to explain verdicts and prison sentence length. Thus, they explore whether a predictive model of sentencing outcome can be constructed from measures of degree centrality, betweenness centrality, and closeness centrality together with graph density and centralization metrics. Their results show that degree centrality explains the likelihood of a guilty or innocent verdict but is less successful in predicting sentence length [1]. What is certain, however, is that these efforts have played a pioneering role in the search for explanatory relationships between sentencing outcome and social network indicators.

Our aim in this study is to pursue Baker and Faulkner's initial findings by assessing variations in criminal justice outcomes in one specific case study. Our analysis expands the repertoire of network measures by integrating a variety of centrality measures that were not assessed by Baker and Faulkner or other researchers since. These variables and the main data source for this case study will be presented in the subsequent section. This data and method description is followed by a series of analyses that demonstrate how positioning in a criminal network is key to understanding how an individual will be reacted to by criminal justice agents.

\section{Centrality measures and criminal behavior}

Centrality is a key concept in social network analysis $[13,34]$ and in academic literature there are different indexes of centrality $[33,35,36]$ which have the common purpose of "quantify an intuitive feeling that in most networks some vertices or edges are more central than others" [37], p. 16. Centrality can be assessed by three types of measures: local, distance, and feedback [38]. Baker and Faulkner [1] already demonstrated criminal justice risks and trade-offs generated by degree centrality, betweenness centrality, and closeness centrality. The additional indicators proposed for this study include out-degree centrality, eigenvector centrality [35], authority centrality and hub centrality [39]. Each of these measures offers a different way of measuring the centrality of an individual in a network.

Degree centrality and its derivative, in-degree and outdegree centrality, are local measures [13]. Degree centrality is a straightforward count of the number of direct contacts that are linked to a node. Out-degree centrality measures the number of direct contacts to whom the node communicates toward (out-flow communication). In-degree centrality measures the number of these direct contacts that communicate toward a node (in-flow communication).

Betweenness centrality and closeness centrality are distance measures [40]. Betweenness centrality measures the extent to which a node mediates relationships between other nodes by its position along the geodesics within the network. A geodesic is the shortest path (or number of degrees) connecting a dyad (a pair of nodes). The greater a node is located along the geodesics in the network, the greater its betweenness centrality. This measure essentially represents the ability of some nodes to control the flow of connectivity (or communication, in this case) within a network. Controlling the flow within the network in this indirect manner is the broker's edge. Closeness centrality is also based on geodesic paths, but unlike betweenness centrality which accounts for mediation within these paths, closeness measures the extent to which a node is in proximity to others. This measure is essentially a calculation of the mean geodesic distance between a node and all other reachable nodes in the network.

Feedback measures in this analysis were accounted for by eigenvector centrality [35], authority centrality and hub centrality [39]. Eigenvector is a derivative of degree centrality in that it measures the extent to which a node is connected to well-connected nodes. Authority centrality and hub centrality are measures that were designed for directed networks. The two are analyzed together and may be described as a mix of in-degree/out-degree centrality and eigenvector centrality. Authority centrality is similar to in-degree centrality in that it measures incoming relationships, while hub centrality is similar to out-degree centrality because it measures outgoing relationships. The difference between the two sets of measures lies in the connectivity of actors that are connected to a node. A node with high authority centrality is one 
that receives a high volume of communications from nodes with high hub centrality. A node with high hub centrality is one that receives a high volume of communications from nodes with high authority centrality. Kleinberg [39] emphasizes the mutually reinforcing relationship between authorities and hubs in that a good hub points to many good authorities, while a good authority points to by many good hubs.

These variables will be used to examine and interpret the patterns surrounding variations in criminal justice outcomes in the Caviar Network. Each set of analysis integrating these variables carry their own rationale. Out-degree centrality measures explain the propagation of messages in a telephone communication network [41,42]. This is important for the present case study since our data are drawn from intercepted communications between criminal network participants. Our hypothesis follows that participants who are more active in this intercepted communication network are more visible (and thus vulnerable) and this will translate into more severe sentencing outcomes.

Betweenness centrality was presented as a more strategic networking pattern [19] in that it represents those participants who are active and pivotal in a network, but less directly involved. Indeed, the broker advantage has been recognized consistent across past research on illegal drug-trafficking [43-46], human smuggling [47-49], stolen-vehicle exportation [50] and general criminal enterprise settings [51-53]. Our hypothesis in the present case study follows that, once a participant is arrested, the brokerage edge is considerably hindered and the key positioning of high-betweenness centrality participants emerges as a heavy burden, thus leading to higher sentencing outcomes.

Baker and Faulkner [1] proposed that closeness centrality is positively related to an unfavorable judicial outcome. However, it should be noted that closeness centrality can also indicate that nodes are distant from a node that has high closeness centrality. In this sense, low closeness centrality helps to identify who was or were the last persons in the flow of communication in a drug trafficking network. We can deduce that an individual with a relatively low closeness centrality may be most vulnerable in the judicial process due to his high visibility and high-risk tasks. Therefore, we expect that the closeness centrality indicator is negatively associated with the likelihood of an unfavorable judicial outcome.

Eigenvector centrality [35,54] represents a participant's connections to well-connected participants. Nodes with the same degree centrality are not necessarily equally central as this will depend on how their contacts are connected. Eigenvector centrality adds this important nuance and captures the problems often associated to proximity to the most visible. In this sense, participants with higher eigenvector centrality should receive more sever sentencing because of their proximity to well-connected individuals in the network who, as hypothesized above, are themselves more likely to receive harsher punishment.

To further nuance the implications of being well connected in a criminal network and being connected to others who are well connected, the authority and hub centrality measures will be introduced to the analysis to assess the reciprocal features that are offered by these variables. Kleinberg argues that these indicators offer "a richer notion of importance, or prominence, [that] contains an intrinsic element of circularity: it arises from the fragile intuition that a node is important if it receives links from other important nodes" [55], p. 611. Our hypothesis therefore posits that a sentencing outcome should penalize more heavily those individuals whose values for these measures reveal a relatively high authority or hub level within the network.

\section{Method}

\section{Case study}

This study is based on the Caviar Network, a hashish and cocaine importation and trafficking network that operated out of Montreal, Canada, during the 1990's $[2,19,56,57]$. The Caviar Network was an international network engaged in hashish and cocaine importation that was the targeted during a 2-year investigation (1994-1996) by the Royal Canadian Mounted Police and the Montreal Police. The strategy adopted by the two forces was unique in that large drug shipments were seize on several occasions ${ }^{\mathrm{a}}$, but no arrests were made until the final phase of the investigation. This enabled investigators to gain detailed knowledge of the criminal behavior and organization of the network participants during the various phases of the investigative process.

\section{Data source}

The source for the network data is the evidence derived from electronic surveillance transcripts that were presented in court during the trials of some of the participants. The more than 1, 000 pages of transcripts released to the public reveal the communication network that existed between network participants. These transcripts were used to create a social network matrix of the drug trafficking operation's communication system during the investigation. The network is valued and directed and is made up of 110 participants. At the end of the investigation, 25 participants were arrested, 22 were charged, and 14 were found guilty. So as not to reveal the identity of the monitored individuals, an identification number was assigned to each (e.g., node $\left.N_{1}, \ldots, N_{110}\right)^{\text {b. }}$. Below is a graph of the Caviar Network ${ }^{\mathrm{c}}$.

Figure 1 shows a sociogram of the Caviar Network, where each node represents an individual member while arrows describe the communications flow. The nodes in 


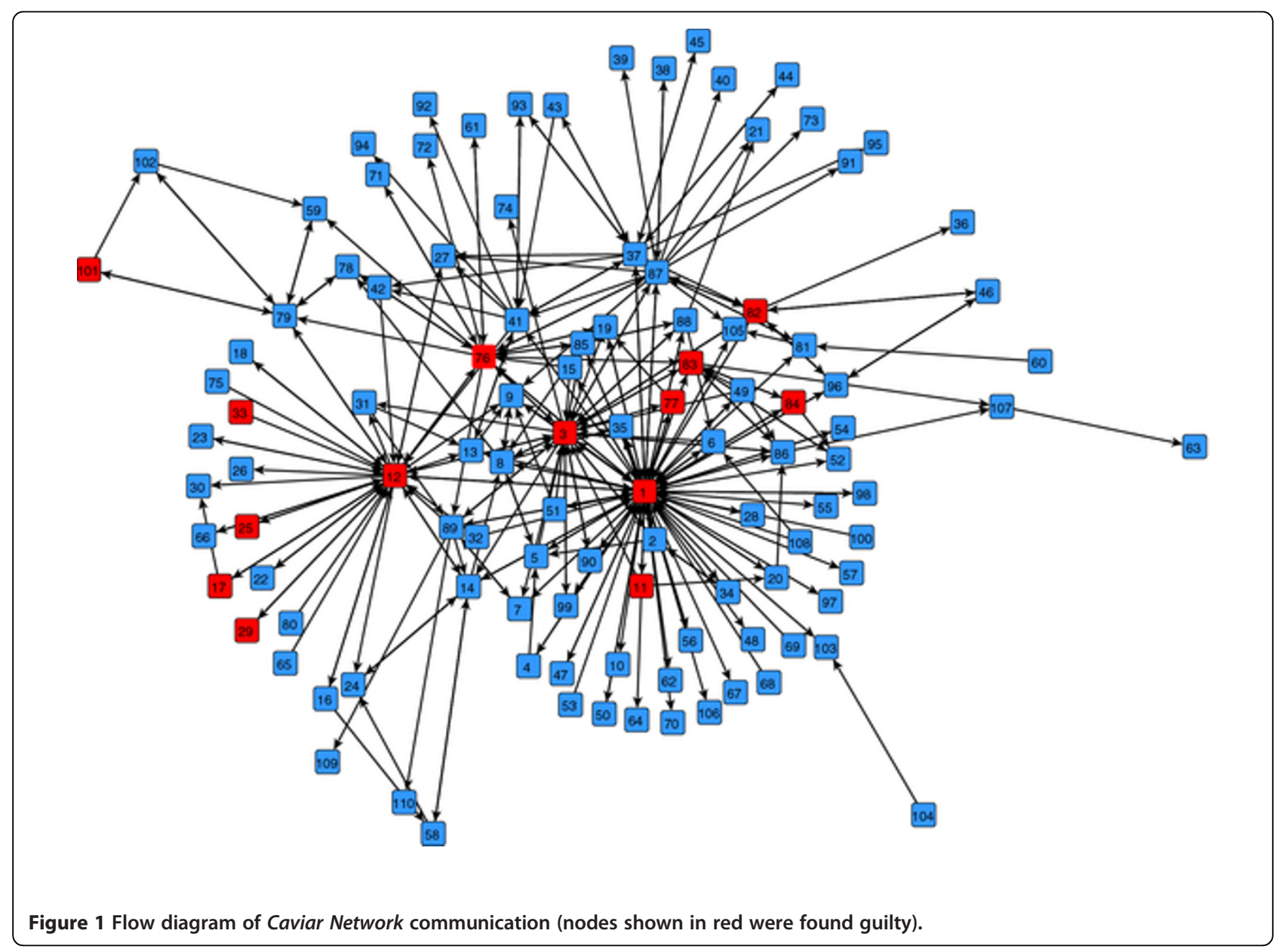

red are the individuals who were found guilty by the courts. As can be seen in the sociogram, there are some nodes that have a clear centrality in the network $\left(N_{1}, N_{2}\right.$ and $N_{3}$ ), but we can also realize that by using only this kind of visual information it is difficult to figure out how the position of each node in the network has a relationship with a coordinated criminal behavior. In fact, some of the participants had heterogeneous and highly specialized roles. For example, individual $N_{1}$ initiated the importation network and was the main coordinator of hashish importations. $N_{12}$ was a Colombian associate who was the main coordinator for cocaine importations. $N_{3}$ was the described as a $N_{1}$ 's lieutenant, but more detailed analysis demonstrated that he was a key liaison between $N_{1}$ and $N_{3}$ [2]. Individual $N_{87}$ was the owner of a legitimate importation enterprise and was one of many participants who did not participate directly in the trafficking activities, but served as key facilitators by supplying legitimate fronts, financial resources, and logistic resources during the importation $\operatorname{activities}^{\mathrm{d}}$ (for this specific analysis, see [56]).

Let us note that the sociogram does not contain data on verbal or written communication, ie., the explicit content of communication between the network's members). Rather, the sociogram shows us the communicative behavior of each individual based on two primitive data types: a) data about who called who and b) data about the number of times where communication occurred between each pair of individuals. Thus, because the social network measure is built from these two simple data types, it is both practical and challenging to analyze whether the centrality measures have the ability to predict the outcomes of a judicial process.

\section{Statistical data analysis}

In this study, two variables are used to indicate the criminal justice outcomes. The first variable is dichotomous and indicates an innocent (coded 0 ) or guilty (coded 1 ) ver$\operatorname{dict}(M=.13, S D=.33)$. The second variable is continuous and indicate the sentence in years which ranged from 0 to 15 years $(M=.86$ years, $S D=2.59)$. The number of cases, minimum and maximum values, means, standard deviations and inter- correlations between outcome measures (variables 1-2) and predictor variables (variables 3-9) are presented in Table 1. 
Table 1 Minima, Maxima, Means, Standard Deviations and Intercorrelations for Outcome Measures and Centrality Measures $(N=110)$

\begin{tabular}{|c|c|c|c|c|c|c|c|c|c|c|c|c|}
\hline Variable & Min. & Max. & $M$ & $S D$ & 2 & 3 & 4 & 5 & 6 & 7 & 8 & 9 \\
\hline \multicolumn{13}{|l|}{ Outcome Measures } \\
\hline 1 Verdict & .00 & 1.00 & .13 & .33 & $.87^{* *}$ & $.28^{* *}$ & $.46^{* *}$ & $.44^{* *}$ & $.43^{* *}$ & $.49^{* *}$ & $.41^{* *}$ & $.31^{* *}$ \\
\hline 2 Year Sentence & .00 & 15.00 & .86 & 2.59 & - & $.26^{* *}$ & $.63^{* *}$ & $.68^{* *}$ & $.59^{* *}$ & $.58^{* *}$ & $.39 * *$ & $.45^{* *}$ \\
\hline \multicolumn{13}{|l|}{ Predictor Variable } \\
\hline 3 Closeness Centrality & .00 & .00 & .00 & .00 & & - & $.23^{*}$ & $.20^{*}$ & $.21^{*}$ & $.24^{*}$ & $.21^{*}$ & .13 \\
\hline 4 Degree Centrality & .11 & 218.00 & 6.87 & 23.07 & & & - & $.94^{* *}$ & $.99^{* *}$ & $.90^{* *}$ & $.42^{* *}$ & $.93^{* *}$ \\
\hline 5 Betweenness Centrality & .00 & $4,663.00$ & 118.09 & 524.68 & & & & - & $.93^{* *}$ & $.73^{* *}$ & $.23^{*}$ & $.88^{* *}$ \\
\hline 6 Out-Degree Centrality & .00 & 109.00 & 2.76 & 11.03 & & & & & - & $.86^{* *}$ & $.30 * *$ & $.96^{* *}$ \\
\hline 7 Eigenvector Centrality & .00 & 1.00 & .04 & .13 & & & & & & - & $.71^{* *}$ & $.80^{* *}$ \\
\hline 8 Authority Centrality & .00 & 1.00 & .04 & .11 & & & & & & & - & .14 \\
\hline 9 Hub Centrality & .00 & 1.00 & .04 & .11 & & & & & & & & - \\
\hline
\end{tabular}

${ }^{*} p<.01$ level $(2-$ tailed $)$.

${ }^{* *} p<.05$ level $(2-$ tailed $)$.

The strategy behind our analysis was to establish which subset of centrality measures had greater predictive ability for sentencing outcomes in the Caviar Network case. More specifically, we set out to determine how the predictive abilities of the measures used in the existing literature (variables 3-5) compared with those discussed in our literature survey above (variables 6-9). There were three steps in the strategy:

1. The first step was to verify whether the degree centrality, betweenness centrality and closeness centrality measures (that is, the measures proposed as sentencing outcome predictors by [1]) were able to predict the outcome of the Caviar Network trial. This was done by conducting an initial logistic regression to predict the verdict of guilt or innocence and an initial multiple linear regression to predict the sentence in years. Both regression models were carried out using the stepwise selection method. The candidate variables in the two regression models were closeness centrality (variable 3 ), degree centrality (variable 4), and betweenness centrality (variable 5). The analysis yielded a) a first model for predicting the verdict (see Table 2), and b) a first model for predicting the sentence length in years (see Table 3).

2. The second step was to select the variables with the best explanatory ability for sentencing outcomes of all the centrality measures studied. This was done using a second logistic regression analysis for predicting the verdict and a second multiple linear regression to predict sentence length. Once again, stepwise selection was employed.

The candidate variables in this second step were all the centrality measures (variables 3-9 in Table 1).
The analysis yielded a) a second model for predicting the verdict (see Table 4), and b) a second model for predicting sentence length (see Table 5).

3 . The third step consisted in making the various comparisons of the model results. The final models for predicting the verdict were compared by observing their respective correct classification percentages. The final models for predicting the sentence (see second Sub-section of Results) were then compared on the basis of the coefficient of determination $\left(R^{2}\right)$ and the error variance $\left(S^{2}\right)$ of the multiple linear regressions.

\section{Results}

The results will be reported as follows. First, we report the summary of logistic regression for predicting the verdict (first Subsection). Second, we report the summary of multiple regressions for predicting the sentence in years (second Subsection).

\section{Logistic regression analysis for centrality measures predicting verdict}

A first logistic regression analysis was conducted to discriminate the verdict, using the Wald statistic to select the significant variables in terms of their prediction ability.

Table 2 Final Regression Model 1 Predicting Verdict

\begin{tabular}{lllllll}
\hline Predictor & $\boldsymbol{B}$ & SE & OR & $\mathbf{9 5 \% C l}$ & $\begin{array}{l}\text { Wald } \\
\text { Statistics }\end{array}$ & $\boldsymbol{p}$ \\
\hline $\begin{array}{l}\text { Closeness } \\
\text { Centrality }\end{array}$ & $-4,910-60$ & 926.76 & .00 & {$[.00,00]$} & 28.08 & $<.001$ \\
$\begin{array}{l}\text { Degree } \\
\text { Centrality }\end{array}$ & .13 & .04 & 1.14 & {$[1.05,1.24]$} & 10.52 & .001 \\
\hline
\end{tabular}

Note. $\mathrm{Cl}=$ confidence interval for odds ratio (OR). 
Table 3 Final Regression Model 1 Predicting Length of Imposed Sentences

\begin{tabular}{llllll}
\hline Predictor & $\boldsymbol{B}$ & $\boldsymbol{S E} \boldsymbol{B}$ & $\boldsymbol{\beta}$ & $\boldsymbol{t}$ & $\boldsymbol{p}$ \\
\hline Betweenness Centrality & $<.01$ & .00 & .63 & 8.95 & $<.001$ \\
Closeness Centrality & $1,118.80$ & 362.14 & .22 & 3.09 & .003 \\
\hline Note. $\boldsymbol{R}^{2}=.51,(N=110, p<.05)$. & & & &
\end{tabular}

The independent variables for this step are betweenness, closeness and degree centrality. The final model used closeness and degree centrality variables to predict the verdict variable (see Table 2 ).

A test of the full model against a constant only model was statistically significant, indicating that the predictors as a set reliably distinguished between guilty and innocent $\left(X^{2} 23.121, p<.003\right.$ with $\left.d f=8\right)$. Nagelkerke's $R^{2}$ of .585 indicated a moderately relationship between prediction and grouping. Prediction success overall was 90.9\% (35.7\% for guilty and $99 \%$ for innocent).

As can be seen in Table 2, the Wald criterion demonstrated that closeness centrality, $p<.001$, and degree centrality, $p<.01$, made a significant contribution to prediction. In the final model the classification was improved by eliminating the regression constant, and the betweenness centrality variable, $p=.655$, was also dropped due to its high residual probability. The odds ratio for closeness centrality is almost $0(O R<.0001)$, indicating that the higher is this measure, the less likely is a guilty verdict. For degree centrality the odds ratio is 1.14, meaning that the higher is this indicator, the more likely is a guilty verdict.

The independent variables in the second regression logistic analysis were the betweenness, closeness and degree centrality indicators, as was the case in the first regression, with the addition of the authority centrality, eigenvector centrality, hub centrality, and out-degree centrality measures. For this logistic regression the Wald statistic was applied in iterative steps to select the significant variables that discriminate the verdict, eliminating the non-significant variables $(p \geq .05)$. The final model used the out-degree centrality and a constant to predict the verdict variable (see Table 4).

A test of the full model against a constant only model was statistically significant, indicating that the predictors as a set reliably distinguished between guilty and innocent $\left(X^{2}=7.228, p<.300\right.$ with $\left.d f=6\right)$. Nagelkerke's $R^{2}$ of .375 implies that the model explained $37.5 \%$ of the relationship

Table 4 Final Regression Model 2 Predicting Verdict

\begin{tabular}{lllllll}
\hline Predictor & B & SE & OR & $\mathbf{9 5 \%} \boldsymbol{C l}$ & $\begin{array}{l}\text { Wald } \\
\text { Statistics }\end{array}$ & $\boldsymbol{p}$ \\
\hline $\begin{array}{l}\text { Out-Degree } \\
\text { Centrality }\end{array}$ & .29 & .08 & 1.34 & {$[1.14,1.57]$} & 12.73 & $<.001$ \\
Constant & -2.85 & .44 & .06 & & 42.94 & $<.001$ \\
\hline
\end{tabular}

Note. $\mathrm{Cl}=$ confidence interval for odds ratio $(O R)$.
Table 5 Final Regression model 2 Predicting length of Imposed Sentences

\begin{tabular}{llllll}
\hline Predictor & $\boldsymbol{B}$ & $\boldsymbol{S E} \boldsymbol{B}$ & $\boldsymbol{\beta}$ & $\boldsymbol{t}$ & $\boldsymbol{p}$ \\
\hline Authority Centrality & $-10,640.00$ & 4.81 & -.46 & -2.21 & .029 \\
Closeness Centrality & $<.01$ & .001 & 1.10 & 8.73 & $<.001$ \\
Eigenvector Centrality & 21.79 & 6.18 & 1.11 & 3.52 & .001 \\
Hub Centrality & -35.82 & 7.02 & -1.27 & -5.11 & $<.001$ \\
\hline
\end{tabular}

Note. $R^{2}=.64,(N=110, p<.05)$.

between prediction and grouping. Prediction success overall was $91.8 \%$ (42.9\% for guilty ${ }^{\mathrm{e}}$ and $99 \%$ for innocent).

As can be seen in Table 4, the Wald criterion demonstrated that only out-degree centrality made a significant contribution to prediction, $p<.001$. In the final model the classification was improved by eliminating the authority centrality variable, $p=.451$, betweenness centrality, $p=.329$, degree centrality, $p=.454$, eigenvector centrality, $p=.854$, hub centrality, $p=.329$, were also dropped due to their high residual probability. Thus, for out-degree centrality the odds ratio is 1.34 , meaning that the higher is this indicator, the more likely is a guilty verdict.

Then, by comparing the percentage correct classification in the first and second logistic regression model, we can report that the second one provides a better prediction of the verdict.

\section{Multiple regression analysis for centrality measures predicting the imposed sentence}

An alpha level of .05 was used. The means, standard deviations and intercorrelations between the variables was presented in Table $1^{\mathrm{f}}$.

A first linear regression was then run to predict the imposed sentence based on the three independent variables: betweenness centrality, closeness centrality and degree centrality. As can be seen in Table 3, the analysis of the data using a linear regression technique revealed that the combined predictors explained $51.5 \%$ of the variance in sentence in years, $R^{2}=.515$, adjusted $R^{2}=.521, F$ $(3.107)=37.95, p<.0001$. Betweenness centrality, $\beta=.003$, $p<.001$, and closeness centrality, $\beta=1,118.800, p<.01$, were significant predictors of the length of the sentence; degree centrality, $\beta=-.003, p=.905$, is not a statistically significant predictor of sentence length. Thus, the regression coefficients indicate that betweenness and closeness centrality increase the length of the sentence.

A second linear regression was run to predict the imposed sentence length. This included the same two sets of centrality measures as independent variables: betweenness, closeness and to degree centrality plus authority centrality, eigenvector centrality, hub centrality, and out-degree centrality. As can be seen in Table 5, the analysis of the data using a linear regression equation revealed that the combined predictors explained $64.4 \%$ 
of the variance in the length of the sentence, $R^{2}=.644$, adjusted $R^{2}=.631, F(4,106)=49.93, p<.0001$. Authority centrality, $\beta=-10,640, p<.05$, betweenness centrality, $\beta=.006, p<.001$, eigenvector centrality, $\beta=21.749, p<.01$, hub centrality, $\beta=-35.822, p<.001$, were significant predictors of the length of the sentence; closeness centrality, $\beta=294.998, p=.431$, degree centrality, $\beta=-.059, p=.596$; out-degree centrality, $\beta=.201, p=.268$, were not statistically significant predictors of the imposed sentence length. Thus, the regression coefficients indicate that betweenness and eigenvector centrality increase the length of the sentence while authority centrality and hub centrality reduce it.

Upon comparing the $R^{2}$ of the first linear regression model (Table 3) with that of the second linear regression model (Table 5), we can then report that the second model provides a better prediction of the sentence in years. In addition, because the error variance in the first regression model, $S^{2}=1.90$, is larger than that of the second regression model, $S^{2}=1.62$, the second model generates errors that are less dispersed. This implies that using the additional set of proposed indicators generates a better prediction.

\section{Discussion and conclusion}

This study has shown that social network centrality indicators have good predictive abilities that can strengthen the efforts of criminologists to explain criminal trial sentencing outcomes using data on the communication behavior of a criminal network. In what follows we offer a number of observations on our findings.

The first point to note is that using a single network analysis centrality measure leads to good verdict predictions (see Table 4). The results were in fact better than the ones generated by the binary regression model reported in [1], although it must be kept in mind that the cases analyzed were different. Our model posits that the out-degree variable explains the finding of innocence or guilt in that it shows that nodes making calls to other nodes are more likely to be classified as guilty, while those found innocent are those who communicate with relatively fewer individuals. It is also true, however, that the model is better at classifying the innocent than the guilty. This result may be due to the fact that the values of the social network indicators are close to 0 , which creates numerical stability problems (on numerical stability in matrices, (see $[58,59]$ ). Furthermore, the number of individuals convicted is low compared to the total number observed (14 out of 110 , or $12.7 \%)$ and a value in the classification is missing, suggesting a separation problem [60]. We therefore conclude that the model predicts guilt or innocence well with a single social network indicator but classifies those found innocent better than those found guilty.
Second, the use of four social network centrality measures generates good predictions of the length of imposed sentences (see Table 5). Certain variables were found to increase or decrease the length of the sentence. Thus, individuals who mediate communication more (betweenness centrality) and those who communicate more with individuals having high centrality levels (eigenvector centrality) receive longer sentences. By contrast, high values for authority or hub centrality decrease sentence length while low values increase it. Individual $N_{1}$, for example, had the highest hub centrality value whereas individual $N_{3}$ displayed the highest authority centrality value, and both were given relatively short sentences.

This last finding might seem to contradict our working hypothesis that higher values for hub and authority centrality should increase sentence length. But it must be kept in mind that in order to be a good hub or authority an individual's node must point to good hubs or good authorities. We suspect that the judicial process imposed light sentences to some nodes that had high hub or authority centralities because the investigative process did not recognize the Caviar Network's complex communication structure. The complexity of the algorithm for computing the hub and authority variables makes it virtually impossible to interpret these variables visually in a graph or sociogram. It appears that the complexity of the network's behavior and authority distribution structures protected certain members while making others vulnerable. In this sense we conclude that our approach explains sentence length based on social network variables.

Although the model generates good predictions, it is noteworthy that the negative weights indicate reductions in sentence length as hub and authority centrality increase, just the opposite of what we would have expected. We believe this occurred because some individuals with high values for these indicators were not punished. This is not to say that the investigation was poorly handled, but nor do we feel obliged to conclude these measures are not good sentence length predictors. A more likely explanation is that the investigators and judges on the case did not have the information generated for this study available to them given the impossibility of obtaining it or observing it directly and were thus not in a position to ensure sentence length was related to network communication behavior. The information they would have needed was derived from a complex algorithmic computation and a sociogram of 110 individuals that is difficult to represent visually. Indeed, at the time of the investigation into the Caviar Network the hub and authority computation algorithm [39] had not yet been formulated. Identifying the authorities in a criminal network is therefore highly important, for without this data the sentences handed down will not be proportional to the actual communication behavior of the Network's member individuals. 
To conclude, the social network indicators used in previous studies already generated good sentencing outcome predictions, the inclusion of additional network centrality measures improves their accuracy. In light of this, three principal considerations should guide future research in this area.

1. The addition of sociodemographic and socioeconomic variables used in the conventional literature to those posited in this study should be explored to determine whether they would improve sentence prediction. In our view, the criminal history variable has already demonstrated its predictive ability regarding sentencing outcome $[3,7,12,61]$. In the Caviar Network case, for example, the node given the longest sentence was individual $N_{12}$, who was condemned to 15 years in prison because of previous convictions.

2. Whereas [1] only included local and distance measures as predictor variables, our results show that local variables function best for predicting the verdict while distance and network feedback variables do a better job of explaining sentence length. Further investigation of sentencing outcomes should include various types of social network measures.

3. There is still no methodology for identifying criminals over time. In the present study we aggregated the 11 matrices describing the behavior of the Caviar Network over a two-year period. The next step would be to carry out a dynamic classification analysis such as the one proposed by [62] to create behavior profiles or patterns for different groups in terms of their social network indicators.

This study has attempted to respond to a number of questions and define new tasks for explaining sentencing outcomes. The ultimate goal is to provide criminologists with valuable feedback for decisions regarding future research and the allocation of resources and effort to issues of public interest. The application of the social network approach requires further study of criminal networks, particularly as regards ethical and legal questions that arise in real-world cases [63]. We agree fully with the observation of McGloin and Kirk that "the use of formal social network analysis is still quite limited in the fields of criminology and criminal justice" [23], p. 222. Greater application of this approach together with the development of new social network measures could provide valuable information on the centrality of individuals in these networks and their network behavior.

\section{Endnotes}

a Morselli [2] reports that these seizures led to a loss of approximately US\$32 million for participants in the network. b Data was organized and analyzed with the Organizational Risk Analyzer (see [64]), a program made available by the CASOS project at Carnegie Mellon University.

c This sociogram was produced using visone software [65]. For more information on graph-layout algorithms and their application in networks social analysis, see the work of [38,66-69].

d Individuals $N_{82}$ to $N_{110}$ represented such facilitators in the network.

e A guilty verdict was correctly predicted for individual $N_{11}$ that the first logistic regression model were unable to classify.

$\mathrm{f}$ The correlation matrix of all variables presented in Table 1 suggests that there is no problem of multicollinearity. As can be note, there is no one variable highly correlated with all others variables.

\section{Competing interests}

The authors declare that they have no competing interests.

\section{Authors' contributions}

$\mathrm{CM}$ carried out the data collection and studied the research domain, $\mathrm{CM}$ and VM designed the research, VM, FC and SL conducted the empirical tests, and VM and SL wrote the paper. All authors read and approved the final manuscript.

\section{Acknowledgement}

We thank all anonymous reviewers for their thorough evaluation and constructive recommendations for improving this manuscript.

\section{Author details}

'School of Criminology, Université de Montréal, C.P. 6128, succursale Centre-ville, Montreal, QC H3C-3 J7, Canada. 'Faculty of Economics and Business, Universidad Diego Portales, Manuel Rodríguez Sur 253, 8370057 Santiago de, Chile. ${ }^{3}$ Department of Management Control, University of Chile, Diagonal Paraguay 257, 8330015, Santiago de, Chile. ${ }^{4}$ Universidad de Valparaíso, Brigadier de La Cruz 1050, 8900183, Santiago de, Chile.

Received: 20 November 2012 Accepted: 27 December 2012 Published: 22 January 2013

\section{References}

1. WE Baker, RR Faulkner, The Social Organization Of Conspiracy: Illegal Networks in the Heavy Electrical Equipment Industry. Am. Sociol. Rev. 58(6), 837-860 (1993)

2. C Morselli, Inside Criminal Networks (Springer Verlag, New York, 2009)

3. SD Bushway, AM Piehl, Judging Judicial Discretion: Legal Factors and Racial Discrimination in Sentencing. Law Soc Rev 35(4), 733-764 (2001)

4. J Dixon, The Organizational Context of Criminal Sentencing. Am. J. Sociol. 100(5), 1157-1198 (1995)

5. MP Harrington, C Spohn, Defining Sentence Type: Further Evidence Against Use of the Total Incarceration Variable. J Res Crime Delinquency 44, 36-63 (2007)

6. S Nicholson-Crotty, KJ Meier, Crime and Punishment: The Politics of Federal Criminal Justice Sanctions. Pol Res Q 56(2), 119-126 (2003)

7. D Steffensmeier, S Demuth, Ethnicity and Sentencing Outcomes in U.S. Federal Courts: Who Is Punished More Harshly? Am. Sociol. Rev. 65(5), 705-729 (2000)

8. L Ellis, KM Beaver, JP Wright, Handbook of Crime Correlates (Academic, San Diego, CA, 2009)

9. R Helms, Modeling the Politics of Punishment: A Conceptual and Empirical Analysis of'Law in Action' in Criminal Sentencing. J Crim Justice 37, 10-20 (2009)

10. C Spohn, D Holleran, The Imprisonment Penalty Paid by Young, Unemployed Black and Hispanic Male Offenders. Criminol 38, 281-306 (2000) 
11. RJ Thomson, MT Zingraff, Detecting Sentencing Disparity: Some Problems and Evidence. Am. J. Sociol. 86(4), 869-880 (1981)

12. SD Bushway, AM Piehl, The Inextricable Link Between Age and Criminal History in Sentencing. Crime \& Delinquency 53, 156-183 (2007)

13. S Wasserman, K Faust, Social Network Analysis: Methods and Applications (Cambridge University Press, Cambridge, 1994)

14. J Sarnecki, Delinquent Networks: Youth Co-Offending in Stockholm (Cambridge University Press, Cambridge, 2001)

15. A Abraham, AE Hassanien, V Snášel, Computational Social Network Analysis: Trends, Tools and Research Advances (Springer, London, 2010)

16. P Carrington, JC Scott, The SAGE Handbook of Social Networks (Sage Publications, London, 2011)

17. JC Scott, Social Network Analysis (Sage Publications, New Park, CA, 1991)

18. P Klerks, The Network Paradigm Applied to Criminal Organizations: Theoretical Nitpicking or a Relevant Doctrine for Investigators? Recent Developments in the Netherlands. Connections 24(3), 53-65 (2001)

19. C Morselli, Assessing Vulnerable and Strategic Positions in a Criminal Network. J Contemp Crim Justice 26(4), 382-392 (2010)

20. MK Sparrow, The Application of Network Analysis to Criminal Intelligence: An Assessment of the Prospects. Soc Netw 13(3), 251-274 (1991)

21. KM Carley, J Reminga, S Borgatti, Destabilizing Dynamic Networks Under Conditions of Uncertainty, in International Conference on Integration of Knowledge Intensive Multi-Agent Systems (IEEE KIMAS, Boston MA, 2003), pp. 121-126

22. VE Krebs, Mapping Networks of Terrorist Cells. Connections 24(3), 43-52 (2002)

23. D McAndrew, The Structural Analysis of Criminal Networks, in The Social Psychology of Crime: Groups, Teams, and Networks, ed. by D Canter, L Alison (Ashgate, Aldershot, 1999), pp. 52-92

24. JJ Xu, H Chen, CrimeNet Explorer: A Framework for Criminal Network Knowledge Discovery. ACM Trans. Inf. Syst. 23(2), 201-226 (2005)

25. KM Carley, JS Lee, D Krackhardt, Destabilizing Networks. Connections 24(3), 31-34 (2001)

26. RS Burt, Applied Network Analysis: A Methodological Introduction (Sage Publications, Beverly Hills, 1983)

27. RS Burt, Brokerage and Closure: An Introduction to Social Capital (Oxford University Press, Oxford, 2005)

28. N Coles, It's Not What You Know, It's Who You Know That Counts: Analyzing Serious Crime Groups as Social Networks. Br. J. Criminol. 4(41), 580-594 (2001)

29. RL Cross, A Parker, The Hidden Power of Social Networks: Understanding How Work Really Gets Done in Organizations (Harvard Business School Press, Boston, 2004)

30. PV Marsden, Brokerage Behavior in Restricted Exchange Networks. Soc Struct. Netw. Anal. 7(4), 341-410 (1982)

31. P Williams, The Nature of Drug-Trafficking Networks. Curr. History 97(618), 154-159 (1998)

32. JM McGloin, DS Kirk, Social Network Analysis, in Handbook of Quantitative Criminology, ed. by AR Piquero, D Weisburd (Springer, New York, 2010), pp. 209-224

33. LC Freeman, Centrality in Social Networks Conceptual Clarification. Soc. Netw. 1(3), 215-239 (1979)

34. G Sabidussi, The Centrality Index of a Graph. Psychometrika 31(4), 581-603 (1966)

35. P Bonacich, Power and Centrality: A Family of Measures. Am. J. Sociol. 92, 11170-1182 (1987)

36. SP Borgatti, Centrality and Network Flow. Social Networks 27, 55-71 (2005)

37. D Koschutzki, KA Lehmann, L Peeters, S Richter, D Tenfelde-Podehl, O Zlotowski, Centrality Indices, in Network Analysis, ed. by U Brandes, T Erlebach (Springer, New York, 2005), pp. 16-61

38. U Brandes, T Erlebach, Network Analysis: Methodological Foundations (Springer Verlag, New York, 2005)

39. JM Kleinberg, Authoritative Sources in a Hyperlinked Environment. J. Assoc Comput. Machinery 46(5), 604-632 (1999)

40. LC Freeman, A Set of Measures of Centrality Based on Betweenness. Sociometry 4(1), 35-41 (1977) [http://www.jstor.org/stable/3033543]

41. MU Khan, SA Khan, Social Networks Identification and Analysis Using Call Detail Records, in Proceedings of the 2nd International Conference on Interaction Sciences: Information Technology, Culture and Human (Association for Computing Machinery, New York, 2009), pp. 192-196
42. C Kiss, A Scholz, M Bichler, Evaluating Centrality Measures in Large Call Graphs, in Proceedings of the 8th IEEE International Conference on ECommerce Technology (IEEE, San Francisco CA, 2006), pp. 47-55

43. FJ Desroches, The Crime that Pays: Drug Trafficking and Organized Crime in Canada (Canadian Scholars' Press, Toronto, ON, 2005)

44. M Natarajan, Understanding the Structure of a Large Heroin Distribution Network: A Quantitative Analysis of Qualitative Data. J. Quant. Criminol. 22(2), 171-192 (2006)

45. G Pearson, D Hobbs, Middle Market Drug Distribution (Home Office Research Study No. 227) (Home Office, London, 2001)

46. D Zaitch, Trafficking Cocaine: Colombian Drug Entrepreneurs in the Netherlands (Springer, The Hague, 2002)

47. E Kleemans, HG Van de Bunt, The Social Organisation of Human Trafficking, in Global Organized Crime: Trends and Developments, ed. by D Siegel, HG van de Bunt, D Zaitch (Kluwer Academic Publishers, Dordrecht, 2003), pp. 97-104

48. S Zhang, Chinese Human Smuggling Organizations: Families, Social Networks, and Cultural Imperatives (Stanford University Press, Stanford, CA, 2008)

49. S Zhang, KL Chin, Enter the Dragon: Inside Chinese Human Smuggling Operations. Criminology 40(4), 737-767 (2002)

50. G Bruinsma, W Bernasco, Criminal Groups and Transnational Illegal Markets. Crime, Law Soc. Change 41, 79-94 (2004)

51. JO Finckenauer, EJ Waring, Russian Mafia in America: Immigration, Culture, and Crime (Northeastern University Press, Boston, 1998)

52. MH Haller, Illegal Enterprise: A Theoretical and Historical Interpretation. Criminology 28, 207-236 (1990)

53. C Morselli, Contacts, Opportunities, and Criminal Enterprise (University of Toronto Press, Toronto, ON, 2005)

54. P Bonacich, Factoring and Weighting Approaches to Status Scores and Clique Identification. J. Math. Sociol. 2, 113-120 (1972)

55. JM Kleinberg, Hubs, Authorities, and Communities. ACM Comput. Surveys (CSUR) 31(4), 1-5 (1999)

56. C Morselli, C Giguère, Legitimate Strengths in Criminal Networks. Crime, Law and Social Change 45(3), 185-200 (2006)

57. C Morselli, K Petit, Law-Enforcement Disruption of a Drug Importation Network. Global Crime 8(2), 109-130 (2007)

58. PG Ciarlet, Introduction to Numerical Linear Algebra and Optimisation (Cambridge University Press, Cambridge, 1989)

59. GH Golub, CF Van Loan, Matrix Computations (Johns Hopkins University Press, Baltimore, 1996)

60. C Zorn, A Solution to Separation in Binary Response Models. Polit. Anal. 13(2), 157-170 (2005)

61. D Steffensmeier, J Ulmer, J Kramer, The Interaction of Race, Gender, and Age in Criminal Sentencing: The Punishment Cost of Being Young, Black, and Male. Criminology 36(4), 763-798 (1998)

62. F Crespo, R Weber, A Methodology for Dynamic Data Mining Based on Fuzzy Clustering. Fuzzy Set Syst. 150(2), 267-284 (2005)

63. C Kadushin, Who Benefits from Network Analysis: Ethics of Social Network Research. Social Networks 27(2), 139-153 (2005)

64. Q Yin, Q Chen, A Social Network Analysis Platform for Organizational Risk Analysis - ORA. In Second International Conference on Intelligent System Design and Engineering Application (IEEE, Sanya, 2012), pp. 760-763

65. U Brandes, D Wagner, Visone: Analysis and Visualization of Social Networks, in Graph Drawing Software, ed. by M Jünger, P Mutzel (Springer Verlag, New York, 2004), pp. 321-340

66. U Brandes, P Kenis, J Raab, Explanation Through Network Visualization. Methodology: European J. Res. Methods Behav. Soc. Sci. 2, 16-23 (2006)

67. W Huang, SH Hong, P Eades, Effects of Sociogram Drawing Conventions and Edge Crossings in Social Network Visualization. J. Graph Algorithms Appl. 11(2), 397-429 (2007)

68. M Huisman, MA Van Duijn, Software for Social Network Analysis, in Models and Methods in Social Network Analysis, ed. by P Carrington, J Scott, S Wasserman (Cambridge University Press, New York, 2005), pp. 270-316

69. D Wagner, Analysis and Visualization of Social Networks, in Proceedings of the 2nd International Conference on Experimental and Efficient Algorithms (WEA'03) (Springer Verlag, New York, 2003), pp. 261-266

doi:10.1186/2190-8532-2-4

Cite this article as: Morselli et al:: Predicting sentencing outcomes with centrality measures. Security Informatics 2013 2:4. 